\title{
Estrutura populacional do siri-baú, Hepatus pudibundus (Herbst 1785) no Norte do Estado do Rio de Janeiro, Sudeste do Brasil
}

\author{
Aline dos Santos Klôh ${ }^{1} \&$ Ana Paula Madeira Di Beneditto ${ }^{1,2}$ \\ ${ }^{1}$ Laboratório de Ciências Ambientais, Centro de Biociências e Biotecnologia - CBB, \\ Universidade Estadual do Norte Fluminense - UENF, \\ Av. Alberto Lamego, 2.000, CEP 28013-602, Campos dos Goytacazes, RJ, Brasil, \\ e-mail: lili_kloh@hotmail.com \\ ${ }^{2}$ Autora para correspondência: Ana Paula Madeira Di Beneditto, e-mail: anapaula@uenf.br
}

KLÔH, A.S. \& DI BENEDITTO, A.P.M. Population structure of the flecked box crab, Hepatus pudibundus (Herbst 1785) in Northern Rio de Janeiro State, Southeastern Brazil. Biota Neotrop. 10(3): http://www. biotaneotropica.org.br/v10n3/en/abstract?short-communication+bn02510032010.

\begin{abstract}
The present study describes the first information about the population structure of the flecked box

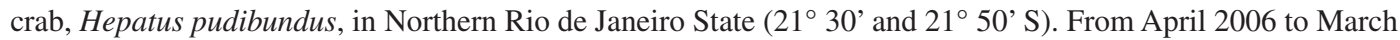
2007 monthly samples $(n=12)$ were conducted using a bottom trawl net aboard local shrimp fishery boats. The specimens were classified by gender and maturity status, measured and weighed. In total, 523 individuals of H. pudibundus were captured: 299 (57.2\%) males and 224 (42.8\%) females. In general, the sex ratio was constant over the studied period, with slight predominance of males. The adult specimens were more numerous than the juveniles' ones. Male specimens were larger and heavier than females, with averages of 46,8 $\pm 9,1 \mathrm{~mm}$ in carapace width and 23,4 $\pm 13,2 \mathrm{~g}$ in weight, compared to 45,3 $\pm 6,6 \mathrm{~mm}$ and $18,8 \pm 7,9 \mathrm{~g}$ for females. The relationships between weight and carapace width were adjusted by the equations $\mathrm{W}=0.0002 . \mathrm{CW} * 3.0391$ $(\mathrm{R} 2=0.97)$ (males) and $\mathrm{W}=0.0003 . \mathrm{CW} * 2.8734(\mathrm{R} 2=0.94)$ (females). The species $H$. pudibundus has little significance to the regional shrimp fishery, but this study indicates its regular occurrence in coastal waters from Northern Rio de Janeiro State.

Keywords: Brachyura, sex-ratio, biometry, artisanal fishery, Rio de Janeiro State.
\end{abstract}

KLÔH, A.S. \& DI BENEDITTO, A.P.M. Estrutura populacional do siri-baú, Hepatus pudibundus (Herbst 1785) no Norte do Estado do Rio de Janeiro, Sudeste do Brasil. Biota Neotrop. 10(3): http://www. biotaneotropica.org.br/v10n3/pt/abstract?short-communication+bn02510032010.

Resumo: O presente estudo descreve as primeiras informações sobre a estrutura populacional do siri-baú, Hepatus pudibundus, na costa Norte do Estado do Rio de Janeiro (213' e $\left.21^{\circ} 50^{\prime} \mathrm{S}\right)$. Entre abril de 2006 e março de 2007 foram realizadas coletas mensais $(n=12)$ com rede de arrasto de fundo em embarcações da pesca camaroneira local. Os espécimes foram classificados quanto ao sexo e maturidade, medidos e pesados. No total, foram capturados 523 indivíduos de H. pudibundus: 299 (57,2\%) machos e 224 (42,8\%) fêmeas. De modo geral, a razão sexual na população se manteve estável ao longo do período de estudo, com ligeiro predomínio de machos. Os espécimes adultos foram mais numerosos do que os juvenis. Os machos foram maiores e mais pesados que as fêmeas, com médias da largura da carapaça de 46,8 $\pm 9,1 \mathrm{~mm}$ e peso de $23,4 \pm 13,2 \mathrm{~g}$, em comparação com 45,3 \pm 6,6 $\mathrm{mm}$ e 18,8 \pm 7,9 $\mathrm{g}$ para as fêmeas. As relações entre peso e largura da carapaça foram ajustadas pelas equações $\mathrm{P}=0,0002 \cdot \mathrm{LC} * 3,0391(\mathrm{R} 2=0,97)$ (machos) e $\mathrm{P}=0,0003 \cdot \mathrm{LC} * 2,8734$ ( $\mathrm{R} 2=0,94$ ) (fêmeas). A espécie H. pudibundus tem pouca representatividade na pesca camaroneira regional, mas o presente estudo indica sua ocorrência regular em associação às águas costeiras do Norte do Estado do Rio de Janeiro.

Palavras-chave: Brachuyra, razão sexual, biometria, pesca artesanal, Estado do Rio de Janeiro. 


\section{Introdução}

O descarte proveniente das capturas acidentais decorrentes de pescarias camaroneiras é uma preocupação mundial, tendo em vista a elevada quantidade de proteína que é desperdiçada (Alverson et al. 1994, Zeler \& Pauly 2005). A baixa seletividade das redes de arrasto com portas ocasiona a captura acidental de muitos invertebrados, tais como esponjas, cnidários, moluscos, anelídeos, crustáceos não alvo e equinodermos (Saila 1983, Svane et al. 2009). No Brasil, a participação de crustáceos braquiúros na fauna acompanhante dessas pescarias é elevada, conforme relatado por Graça-Lopes et al. (2002), Severino-Rodrigues et al. (2002), Branco \& Fracasso (2004) e Costa \& Di Beneditto (2009), mas esses organismos tem baixo valor comercial ou são descartados após a captura. Hepatus pudibundus (Herbst 1785), popularmente conhecido como siri-baú, é o único representante da família Calappidae nessa fauna acompanhante (Fracasso \& Branco 2005, Severino-Rodrigues et al. 2002, Costa \& Di Beneditto 2009). Keunecke et al. (2007) apontaram para uma sobrepesca deste crustáceo entre $23^{\circ} 18^{\prime}$ e $23^{\circ} 58^{\prime} \mathrm{S}$, em decorrência da captura acidental pela pesca camaroneira.

A espécie H. pudibundus se distribui no Oceano Atlântico Sul, do Sul dos Estados Unidos ao Sul do Brasil, e da Guiné à África do Sul, com preferência por fundos de lama e/ou areia e lama, desde águas rasas até $160 \mathrm{~m}$ de profundidade (Melo 1996). Esse calapídeo possui longevidade curta, em torno de 1 ano e 7 meses; alcança a maturidade sexual com tamanho corporal entre 32 e $36 \mathrm{~mm}$ de largura da carapaça; se reproduz continuamente ao longo do ano e, quando adulto, atinge dimensões que variam entre 60 e $80 \mathrm{~mm}$ de largura da carapaça (Mantelatto \& Fransozo 1992, 1994, Reigada \& NegreirosFransozo 1999, Fracasso \& Branco 2005, Keunecke et al. 2007). Estudo realizado por Mantelatto \& Petracco (1997) aponta para um hábito alimentar onívoro, com dieta composta principalmente por sedimento, moluscos, crustáceos peneídeos e peixes.

A captura de $H$. pudibundus pela pesca camaroneira praticada na costa Norte do Estado do Rio de Janeiro é reportada pelo presente estudo, e as primeiras informações sobre a sua estrutura populacional na região são apresentadas. Dessa forma, pretende-se ampliar o entendimento sobre a biologia da espécie no Sudeste do Brasil, comparando as informações obtidas com os dados de outras populações previamente estudadas.

\section{Material e Métodos}

A fauna acompanhante do camarão sete barbas (Xiphopenaeus kroyeri Heller 1862) foi coletada mensalmente $(n=12)$ entre abril de 2006 e março de 2007. Os arrastos foram realizados ao longo da área de pesca das embarcações sediadas no porto de Atafona, Norte do Estado do Rio de Janeiro, entre $21^{\circ} 30^{\prime}$ e $21^{\circ} 50^{\prime} \mathrm{S}$, a partir de menos de uma (1) até 3 milhas náuticas de distância da linha de costa, em profundidades que variaram de 10 a $15 \mathrm{~m}$ (Figura 1). $\mathrm{O}$ esforço de pesca em cada amostragem foi de três a quatro horas, com a embarcação se deslocando em média a $5 \mathrm{~km} / \mathrm{h}$. A rede de arrasto de fundo utilizada media $10 \mathrm{~m}$ de comprimento, $6 \mathrm{~m}$ de abertura horizontal e $30 \mathrm{~mm}$ de malha (esticada entre nós não adjacentes) na sua porção terminal.

Os espécimes de $H$. pudibundus foram separados dos demais organismos após a captura, no entreposto de pesca. Os indivíduos foram avaliados quanto ao sexo e o reconhecimento das fêmeas ovígeras foi realizado por meio da observação dos somitos abdominais. A proporção sexual foi determinada mensalmente e o teste Qui-quadrado aplicado para verificar a significância das diferenças ao longo dos meses de coleta $(p \leq 0,05)$.
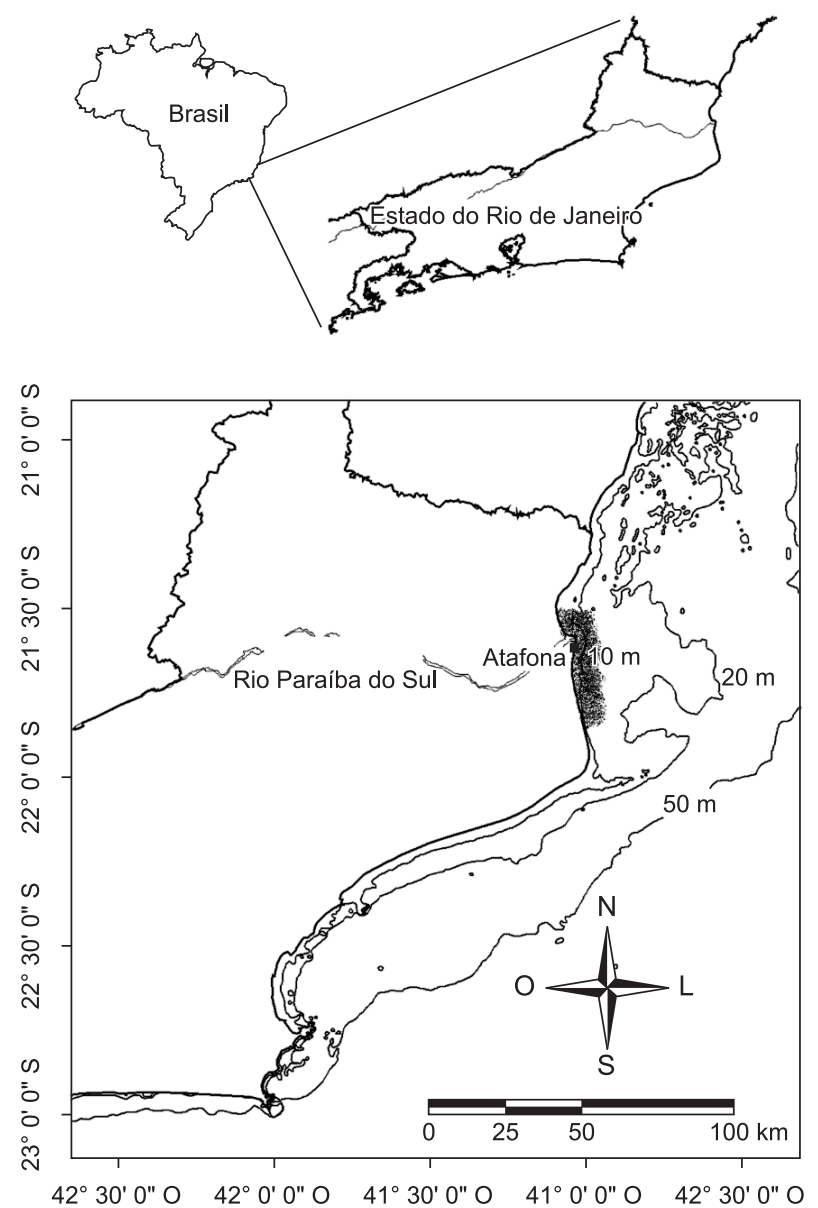

Figura 1. Mapa indicando o Estado do Rio de Janeiro, o porto de Atafona e a área onde os espécimes de Hepatus pudibundus (Herbst 1785) foram coletados.

Figure 1. Map showing Rio de Janeiro State, Atafona harbor and the area where Hepatus pubibundus (Herbst 1785) specimens were collected.

Cada indivíduo foi medido quanto à largura da carapaça (LC, $1 \mathrm{~mm})$ e o peso total $(0,1 \mathrm{~g})$ foi tomado apenas para aqueles que apresentavam os cinco pares de pereiópodos. Foram considerados como adultos todos os indivíduos com LC igual ou maior que $36 \mathrm{~mm}$ (Mantelatto \& Fransozo 1994, Reigada \& Negreiros-Fransozo 1999, Fracasso \& Branco 2005).

A distribuição de frequência da LC considerou machos e fêmeas em separado, agrupando-se os espécimes em intervalos de $5 \mathrm{~mm}$. A relação entre o peso e a LC foi ajustada em separado para machos e fêmeas, e representada pela equação $\mathrm{P}=a$. $\left(\mathrm{LC}^{*} b\right)$, onde $\mathrm{P}$ é o peso em gramas.

\section{Resultados}

Foram coletados 523 indivíduos (299 machos, 57,2\% e 224 fêmeas, 42,8\%), correspondendo a 7,7\% do número total de crustáceos braquiúros registrados na região $(n=6.833$ indivíduos). A espécie esteve presente em $75 \%$ das amostragens, com ausência em janeiro, agosto e setembro. Apenas seis fêmeas ovígeras foram registradas entre abril, maio e outubro (Tabela 1 ).

A proporção sexual durante os meses de coleta manteve-se estável, com exceção de maio, quando os machos foram coletados em maior número (Tabela 1). Foram observadas diferenças significativas em relação às classes de maturidade ao longo do ano, para ambos os sexos, com os indivíduos adultos superando os jovens na malha amostral (Tabela 1). 
Tabela 1. Número de Hepatus pudibundus (Herbst 1785) coletados no Norte do Estado do Rio de Janeiro em cada mês, considerando sexo e classe de maturidade, e comparações entre a proporção sexual e as classes de maturidade pelo teste Qui-quadrado.

Table 1. Hepatus pudibundus (Herbst 1785) monthly collected in Northern Rio de Janeiro State, considering sex and maturity class, and comparisons between sex ratio and maturity class through Qui-square test.

\begin{tabular}{|c|c|c|c|c|c|c|c|}
\hline \multirow{3}{*}{ Meses } & \multicolumn{2}{|c|}{$\operatorname{Machos}(n=299)$} & \multicolumn{3}{|c|}{ Fêmeas (n = 224) } & \multicolumn{2}{|c|}{ Qui-quadrado } \\
\hline & \multirow{2}{*}{$\begin{array}{c}\text { Jovens } \\
(\mathbf{n}=\mathbf{3 5})\end{array}$} & \multirow{2}{*}{$\begin{array}{l}\text { Adultos } \\
(n=264)\end{array}$} & \multirow{2}{*}{$\begin{array}{c}\text { Jovens } \\
(\mathrm{n}=13)\end{array}$} & \multicolumn{2}{|c|}{ Adultas } & \multirow{2}{*}{$\begin{array}{c}\text { Proporção } \\
\text { sexual }\end{array}$} & \multirow{2}{*}{$\begin{array}{c}\text { Classe de } \\
\text { maturidade }\end{array}$} \\
\hline & & & & Não ovígeras $(n=205)$ & Ovígeras $(n=6)$ & & \\
\hline abr./06 & 1 & 28 & 0 & 20 & 3 & 0,7 & $48,1 *$ \\
\hline maio/06 & 25 & 73 & 6 & 41 & 2 & $16,3^{*}$ & $49,1 *$ \\
\hline jun./06 & 0 & 2 & 0 & 2 & 0 & 0 & $4,0^{*}$ \\
\hline jul./06 & 1 & 24 & 2 & 14 & 0 & 1,9 & $29,8 *$ \\
\hline ago./06 & 0 & 0 & 0 & 0 & 0 & - & - \\
\hline set./06 & 0 & 0 & 0 & 0 & 0 & - & - \\
\hline out./06 & 7 & 82 & 4 & 71 & 1 & 1 & $123,9 *$ \\
\hline nov./06 & 0 & 4 & 0 & 1 & 0 & 1,8 & $5,0^{*}$ \\
\hline dez./06 & 0 & 27 & 0 & 21 & 0 & 0,8 & $48,0 *$ \\
\hline jan./07 & 0 & 0 & 0 & 0 & 0 & - & - \\
\hline fev./07 & 1 & 20 & 1 & 30 & 0 & 1,9 & $44,3^{*}$ \\
\hline mar./07 & 0 & 4 & 0 & 5 & 0 & 0,1 & $9,0^{*}$ \\
\hline
\end{tabular}

$* p \leq 0,05$.

A largura da carapaça dos machos media entre 23 e $68 \mathrm{~mm}$ $(46,8 \pm 9,1 \mathrm{~mm})$ e a das fêmeas entre 28 a $62 \mathrm{~mm}(45,3 \pm 6,6 \mathrm{~mm})$. Quanto ao peso, os machos também apresentaram maiores dimensões, variando de 2,3 a 67,7 $\mathrm{g}(23,4 \pm 13,2 \mathrm{~g})$, enquanto as fêmeas ficaram entre 4,3 e 46,6 g (18,8 \pm 7,9 g) (Figura 2). A relação entre o peso e a LC revelam crescimento aproximadamente isométrico para os machos $(\mathrm{P}=0,0002$. $(\mathrm{LC} * 3,0391) ; \mathrm{R} 2=0,97 ; \mathrm{n}=235)$ e alométrico negativo para as fêmeas $(\mathrm{P}=0,0003$. $(\mathrm{LC} * 2,8734)$; $\mathrm{R} 2=0,94$; $\mathrm{n}=184$ ) (Figura 2).

\section{Discussão}

O presente estudo revela um padrão de ocorrência regular de H. pudibundus na região, confirmando a sua preferência por águas costeiras com fundo de lama ou de areia e lama, que caracterizam a área de estudo. Investigações sobre populações da espécie ao longo do litoral brasileiro apontam para o predomínio das fêmeas em relação aos machos (Mantelatto et al. 1995, Reigada \& Negreiro-Fransozo 1999, Fracasso \& Branco 2005, Keunecke et al. 2007). Segundo Haley (1979), as diferenças inter sexuais quanto ao crescimento, mortalidade e padrão de migração podem influenciar na proporção sexual de crustáceos nas áreas de ocorrência. No presente estudo, entretanto, a proporção sexual manteve-se estável ao longo da maioria dos meses de coleta. Isso pode ser uma característica da população local de $H$. pudibundus ou refletir a estrutura desta população no período em que a amostragem foi realizada.

A baixa representatividade de fêmeas ovígeras na malha amostral pode estar relacionada a sua migração para áreas abrigadas devido à pressão pesqueira constante e/ou preferência diferenciada em relação à profundidade, temperatura e textura do sedimento (Mantelatto \& Fransozo 1999). No caso dos juvenis de ambos os sexos, a baixa seletividade da malha da rede de arrasto pode explicar a sua escassez na amostragem, conforme já proposto por Branco \& Fracasso (2004). Essa mesma característica foi verificada em outras áreas do litoral
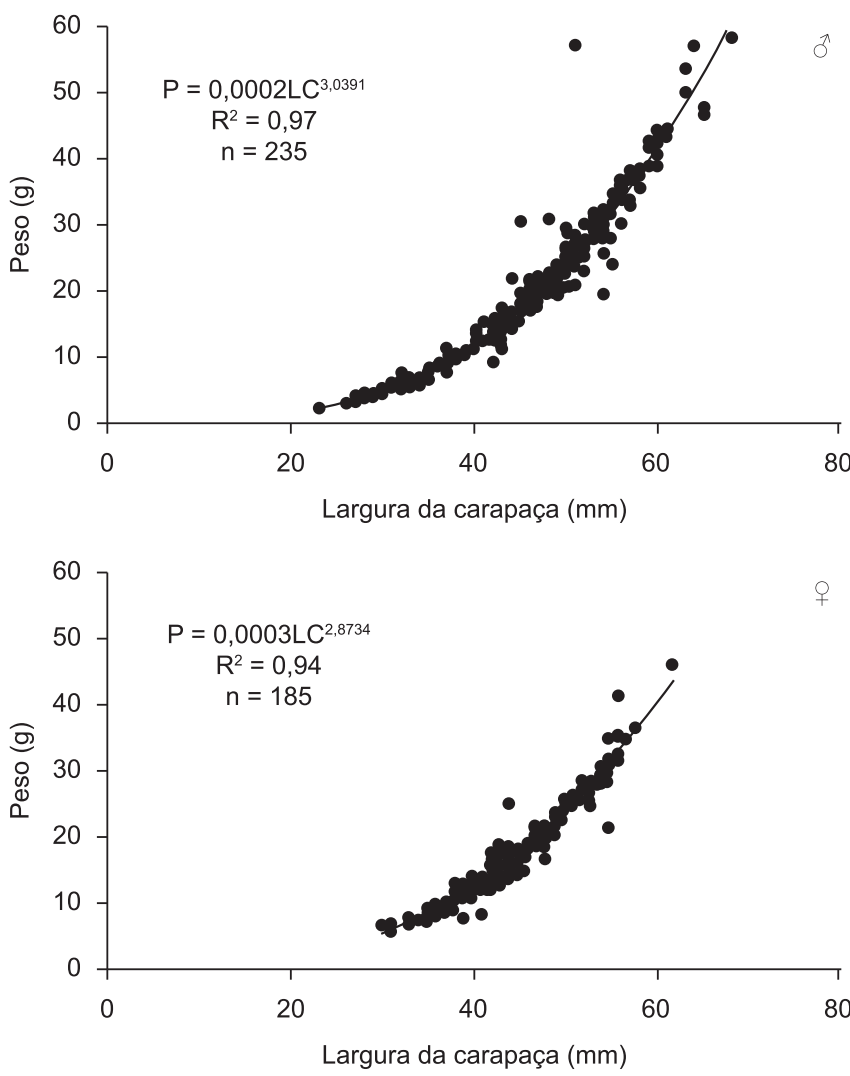

Figura 2. Relação entre peso (g) e largura da carapaça (mm) de machos e fêmeas de Hepatus pudibundus (Herbst 1785) no Norte do Estado do Rio de Janeiro.

Figure 2. Relationship between weight (g) and carapace width (mm) of males and females of Hepatus pudibundus (Herbst 1785) in Northern Rio de Janeiro State. 
brasileiro onde a espécie $H$. pudibundus foi avaliada, registrando-se sempre um percentual mais elevado de indivíduos adultos (Mantelatto et al. 1995, Reigada \& Negreiros-Fransozo 1999, Fracasso \& Branco 2005, Keunecke et al. 2007). Adicionalmente, a segregação espacial entre jovens e adultos da espécie não pode ser descartada como explicação para tal fato, mas são necessários estudos específicos para elucidar tal condição.

A largura máxima da carapaça de machos e fêmeas de H. pudibundus registrados no Norte do Estado do Rio de Janeiro foi cerca de 10-15\% inferior ao reportado para outras áreas do Sudeste e Sul do Brasil (Mantelatto et al. 1995, Fracasso \& Branco 2005, Keunecke et al. 2007) (Tabela 2). As diferenças podem ter relação com fatores não mutuamente excludentes, tais como: i) taxa de crescimento e tamanho corporal distintos devido a variações latitudinais e de temperatura entre as regiões geográficas, ii) variações na pressão exercida pela pesca camaroneira sobre as populações deste braquiúro e iii) variações no esforço de amostragem, incluindo áreas de coleta e tamanho de malha das redes utilizadas. Em relação ao primeiro fator, é esperado que populações de uma dada espécie de crustáceo que se distribuem em áreas de menores latitudes e temperaturas elevadas sejam formadas por indivíduos de tamanho corporal menor em comparação àquelas que ocorrem em latitudes maiores e, consequentemente, temperaturas mais baixas (Hartnoll 1982). Essa condição foi confirmada pelos dados do presente estudo e pela comparação com informações da literatura (Tabela 2). Em relação à pressão pesqueira, a retirada contínua de indivíduos de uma mesma classe de tamanho pode alterar a estrutura da população ao longo do tempo. Entretanto, uma série temporal mais longa e comparações entre o esforço de pesca praticado nas áreas de ocorrência da espécie são necessárias para a confirmação dessa condição.

Em geral, os machos apresentaram tamanho corporal e peso maiores que as fêmeas, o que confere com o padrão descrito na literatura para a maior parte dos braquiúros (Hartnoll 1982). Mantelatto \& Fransozo (1994) e Reigada et al. (1994) levantam fatores explicativos para essa diferença em $H$. pudibundus: i) os quelípodos dos machos são maiores e mais pesados; ii) a pressão de seleção reprodutiva atua de modo favorável em relação aos machos maiores, que por sua vez estariam deixando mais descendentes; e iii) a fêmea, após atingir a maturidade sexual, investe mais no metabolismo reprodutivo e na maturação das gônadas do que em seu crescimento.

A relativa isometria verificada na relação entre peso e LC de machos da espécie indica aumento proporcional dessas variáveis com o desenvolvimento do indivíduo. No caso das fêmeas, a relação alométrica negativa indica que o ganho de biomassa diminui com a maturidade do animal, o que pode ser explicado pela redução no investimento somático após atingirem a maturidade (Reigada et al. 1994). O mesmo resultado foi obtido por Mantelatto \& Fransozo (1992), em estudo conduzido no litoral Norte do Estado de São Paulo (Tabela 2). No entanto, Fracasso \& Branco (2005) e Keunecke et al. (2007) reportam relações biométricas distintas das citadas acima (Tabela 2). Apesar das diferenças, todos os autores verificaram que o coeficiente de alometria dos machos é maior, indicando ganho de biomassa superior ao das fêmeas ao longo do crescimento. As variações entre os estudos podem refletir as características biométricas das populações de $H$. pudibundus ao longo das áreas de distribuição, ou apenas diferenças temporais na ontogenia dos indivíduos que compõe essas populações. Como todos os estudos supracitados e a presente abordagem foram realizados por período de 12 meses, sugere-se a condução de uma série temporal de amostragem mais longa para confirmação dessas relações biométricas.

No Norte do Estado do Rio de Janeiro e em outras regiões do Brasil, a espécie $H$. pubibundus é registrada como fauna acompanhante da pesca camaroneira (Severino-Rodrigues et al. 2002, Branco \& Fracasso 2004, Costa \& Di Beneditto 2009). De acordo com os autores supracitados e os dados do presente trabalho, a sua participação numérica não ultrapassa $10 \%$ do total de braquiúros registrados nesse tipo de captura acidental. A baixa representatividade de $H$. pudibundus em relação às espécies de braquiúros que estão em simpatria parece indicar que um processo de exclusão competitiva está ocorrendo. Adicionalmente, a ação contínua da atividade de pesca camaroneira em regiões costeiras pode influenciar no tamanho e na estrutura das populações de braquiúros a partir da retirada sistemática de indivíduos, levando a alterações na taxa de recrutamento e no tamanho populacional. Diante disso, recomenda-se a continuidade de amostragens dessa natureza para avaliação do impacto dessa atividade pesqueira sobre as populações de $H$. pudibundus e dos demais braquiúros ao longo de suas áreas de ocorrência.

Tabela 2. Comparação entre largura da carapaça e relações biométricas de Hepatus pudibundus (Herbst 1785) no Sudeste e Sul do Brasil.

Table 2. Comparison between carapace width and biometric relationships of Hepatus pudibundus (Herbst 1785) in Southeastern and South Brazil.

$\begin{array}{cccc}\text { Local } & \begin{array}{l}\text { Largura da carapaça }(\mathrm{mm}) \\ \text { (mín-máx; média } \pm \mathrm{dp})\end{array} & \mathbf{P}=\mathbf{a} .(\mathrm{LC} * \mathbf{b}) & \text { Referência }\end{array}$

Rio de Janeiro

$\left(21^{\circ} 30^{\prime}-21^{\circ} 50^{\prime} \mathrm{S}\right)$

Rio de Janeiro e São Paulo (23ำ $\left.18^{\prime}-23^{\circ} 58^{\prime} \mathrm{S}\right)$

São Paulo

$\left(23^{\circ} 31^{\prime} \mathrm{S}\right)$

São Paulo

$\left(23^{\circ} 31^{\prime} \mathrm{S}\right)$

Santa Catarina

$\left(26^{\circ} 42^{\prime}-26^{\circ} 46^{\prime} \mathrm{S}\right)$
ก) $23-68 ; 46,8 \pm 9,1$

ㅇ $28-62 ; 45,3 \pm 6,6$

đ̊ 83,1 (máx)

† 67,1 (máx)

$-$

○ $13,4-78,2 ; 53,2 \pm 10,5$
o $18,3-66,4 ; 46,7 \pm 7,8$
ภิ $\mathrm{P}=2.10-4 .\left(\mathrm{LC}^{*} 3,0391\right)(\mathrm{n}=235)$

우 $\mathrm{P}=3.10-4$. $(\mathrm{LC} * 2,8734)(\mathrm{n}=185)$

đ $\ln \mathrm{P}=3,38 \cdot \operatorname{lnLC}-10,22(\mathrm{n}=408)$

우 $\ln \mathrm{P}=3,17 \cdot \ln L \mathrm{C}-9,41(\mathrm{n}=524)$

๙ิ $\mathrm{P}=1,95 \cdot 10-4 .(\mathrm{LC} * 3,01)(\mathrm{n}=244)$

ㅇ $\mathrm{P}=3,76 \cdot 10-4 .(\mathrm{LC} * 2,84)(\mathrm{n}=380)$
Presente estudo

Keunecke et al. (2007)

Mantelatto \& Fransozo (1992)

Mantelatto et al. (1995) đิ 10-80 (mín-máx)

† 10-70 (mín-máx)
त $\mathrm{P}=0,2237 .(\mathrm{LC} * 2,9405)(\mathrm{n}=473)$

O $\mathrm{P}=0,2703 .(\mathrm{LC} * 2,8312)(\mathrm{n}=846)$
Fracasso \& Branco (2005) 


\section{Agradecimentos}

Aos pescadores do porto de Atafona e à técnica de campo Silvana Ribeiro Gomes pelo auxílio na coleta dos espécimes de Hepatus pudibundus. Ao IBAMA pela concessão da licença de coleta permanente de material zoológico n ${ }^{\circ}$ 16401-1. A.S. Klôh agredece à FAPERJ pela concessão de bolsa de Iniciação Científica. A.P.M. Di Beneditto agradece à FAPERJ (E-26/110.286/07e E-26/103.038/08) e ao CNPq (305160/06-0 e 470021/08-0) pela concessão de bolsa de pesquisa e auxílio financeiro.

\section{Referências Bibliográficas}

ALVERSON, D.L., FREEBERG, M.H., POPE, J.G. \& MURAWISK, S.A 1994. A global assessment of fisheries bycatch and discards. FAO Fisheries Technical Paper, 339.

BRANCO, J.O. \& FRACASSO, H.A.A. 2004. Ocorrência e abundância da carcinofauna acompanhante na pesca do camarão sete-barbas, Xiphopenaeus kroyeri Heller (Crustácea, Decapoda), na Armação do Itapocory, Penha, Santa Catarina, Brasil. Rev. Bras. Zool. 21(2):295-301.

COSTA, I.D. \& DI BENEDITTO, A.P.M. 2009. Caracterización preliminary de los invertebrados bentónicos capturados accidentalmente em la pesca de camarones en el Norte del estado de Río de Janeiro, Sudeste de Brasil. Lat. Am. J. Aquatic. Res. 37:259-264.

FRACASSO, H.A.A. \& BRANCO, J.O. 2005. Estrutura populacional de Hepatus pudibundus (Herbst,1785) (Crustacea, Decapoda) na Armação de Itapocoroy, Penha, Santa Catarina, Brasil. Rev. Bras. Zool. 22(2):342-348.

GRAÇA-LOPES, R., TOMÁS, A.R.G., TUTUI, S.L.S., SEVERINORODRIGUES, E. \& PUZZI, A. 2002. Fauna acompanhante da pesca camaroneira no litoral do Estado de São Paulo, Brasil. Bol. Inst. Pesca 28:173-188.

HALEY, S.R. 1979. Sex ratio as a function of size in Hippa pacifica Dana (Crustacea, Anomura, Hippidae): a test of the sex reversal and differential growth rate hypothesis. Am. Nat. 113:391-397.

HARTNOLL, R.G. 1982. Growth. In The Biology of Crustacea (D. Bliss, ed.). Academic Press, New York, p.111-185.

KEUNECKE, K.A., D'INCAO, F. \& FONSECA, D.B. 2007. Growth and mortality of Hepatus pudibundus (Crustacea:Calappidae) in Southwestern Brasil. J. Mar. Biol. Ass. U.K. 87:885-891.
MANTELATTO, F.L.M. \& FRANSOZO, A. 1992. Relação peso/largura da carapaça do caranguejo Hepatus pudibundus (Herbst, 1785) (Crustacea, Decapoda, Calappidae) na região de Ubatuba, SP, Brasil. Arq. Biol. Tec. 35(4):719-724.

MANTELATTO, F.L.M. \& FRANSOZO, A. 1994. Crescimento relativo e dimorfismo sexual em Hepatus pudibundus (Herbst, 1785) (Decapoda, Brachyura) no litoral Norte paulista. Pap. Avul. Zool. 39(4):33-48.

MANTELATTO, F.L.M. \& FRANSOZO, A. 1999. Reproductive biology and moulting cicle of the crab Callinectes ornatus (Decapoda, Portunidae) from the Ubatuba region, São Paulo, Brazil. Crustaceana 72(1):63-76.

MANTELATTO, F.L.M. \& PETRACCO, M. 1997. Natural diet of the crab Hepatus pudibundus (Brachyura, Calappidae) in Fortaleza bay, Ubatuba (SP) Brazil. J. Crust. Biol. 17(3):440-446.

MANTELATTO, F.L.M., FRANSOZO, A. \& NEGREIROS-FRANSOZO, M.L. 1995. Population structure of Hepatus pudibundus (Decapoda: Calappidae) in Fortaleza Bay, Brasil. Rev. Biol. Trop. 43:265-270.

MELO, G.A.S. 1996. Manual de identificação dos Brachyura (caranguejos e siris) do litoral brasileiro. Plêiade/FAPESP, São Paulo.

REIGADA, A.L.D. \& NEGREIROS-FRANSOZO, M.L. 1999. Maturidade sexual em Hepatus pudibundus (Decapoda, Brachyura, Calappidae). Iheringia, Sér. Zool. 86:159-164.

REIGADA, A.L.D., NEGREIROS-FRANSOZO, M.L. \& MANTELATTO, F.L.M. 1994. Avaliação do tamanho dos quelípodos de Hepatus pudibundus (Herbst, 1785) (Crustacea, Decapoda, Calappidae), em relação ao sexo e à maturação. Arq. Biol. Tec. 37(4):792-807.

SAILA, S.B. 1983. Importance and assessment of discards in commercial fisheries. Food and Agriculture Organization of the United Nations. Fisheries Circular, 765.

SEVERINO-RODRIGUES, E., GUERRA, D.S.F. \& GRAÇA-LOPES, R. 2002. Carcinofauna acompanhante da pesca dirigida ao camarão-setebarbas (Xiphopenaeus kroyeri) desembarcada na praia do Perequê, Estado de São Paulo, Brasil. Bol. Inst. Pesca. 28:33-48.

SVANE, I., HAMMETT, Z. \& LAUER, P. 2009. Impacts of trawling on benthic macro-fauna and-flora of the Spencer Gulf prawn fishing grounds. Estuar. Coast. Mar. Sci. 82:621- 631.

ZELER, D. \& PAULY, D. 2005. Good news, bad news: global fisheries discards are declining, but so are total catches. Fish Fish. 6:156-159.

Recebido em 20/04/2010 Versão reformulada recebida em 22/06/2010 Publicado em 05/08/2010 